\title{
On Congestion in Mission Critical Networks
}

\author{
Ali Tizghadam \\ School of Electrical and Computer Engineering \\ University of Toronto, Toronto, Canada \\ Email: ali.tizghadam@utoronto.ca
}

\author{
Alberto Leon-Garcia \\ School of Electrical and Computer Engineering \\ University of Toronto, Toronto, Canada \\ Email: alberto.leongarcia@utoronto.ca
}

\begin{abstract}
This paper studies the effect of congestion in mission critical networks and explores the network topologies which are appropriate for highly congested networks. Theoretical concepts of "graph theory" are the deriving points to understand the critical situations in telecommunication networks and help find the best topologies based on the network conditions. Towards this end, we evaluate the "betweenness centrality" of network topologies. We assign a robustness index to each network using the result of our evaluation. This "Network Robustness Index" (NRI) can be used to rank different networks based on their robustness to the congestion. One would be able to choose appropriate network topologies in different situations based on the value of NRI. NRI guides the network evolution towards a robust topology which is resilient against congestion. The evolution is in the direction of decreasing the chance of congestion.
\end{abstract}

Index Terms-Robustness, Graph-Theory, Betweenness, Congestion, Network Design Problem.

\section{INTRODUCTION}

One of the crucial problems in network management is to make the whole communication system "robust" to possible changes in different parameters of the network due to uncertainties. This is specially important in Mission Critical Networks (MCN), where these unexpected changes in system parameters may cause disaster. First we give our definition of robustness. There are three major type of changes that may affect the performance of the network [1]:

1. Network topology and connectivity. This includes changes in capacity of the links.

2. Community of interest.

3. Traffic Matrix.

Throughout this paper, we call a "network topology" robust if it can resist against uncertainties which are the result of changes in topology, traffic or community of interest. Our stress in this paper is in traffic surges that might guide network to the congestion mode.

In [1] the goal was to find a robust routing plan for the core network that allows the network service provider to manage the assignment of flows to the paths primarily at the edge of the core network and obtain close to maximum throughput. The network topology was already designed and established, only incremental changes due to failures could happen. In contrast, this work is dedicated to the initial network design problem considering the congestion minimization as the goal. Network design problem (NDP) is a well studied subject In communication systems. We believe that the network design including topology design and capacity assignment is an integrated part of the network management system and should evolve according to the dynamic changes in the network parameters and conditions.

We give the details for topology design considering the congestion minimization as the goal while the capacity is assumed to be known. We assume that every single node can be modeled with a capacitated queue. Obviously if the external input rate of the traffic starts growing, after a while the input rate would be more than the service rate of the queue and this makes accumulation in the queue buffers and finally the congestion is happening. In this research we are trying to explore the range of network topologies, which are appropriate for this queue-based congestion effect. We investigate the properties of appropriate topologies using the metrics from graph-theory.

The rest of this paper is organized as follows. Section II reviews previous works on network design and robustness problem in networks. In section III the network design problem is investigated.Further, details of our analytical results and the design guidelines for topology selection and capacity allocation. Simulation results and validation are provided in section IV and the paper is concluded in sectionV.

\section{PREVIOUS WORK}

A wealth of literature is available about network robustness and different aspects of it. In [2] some facts from graphtheory are reviewed which are important for the development of robust network topologies.

Robustness to the traffic uncertainty is investigated from different standpoints. In [3] a framework for robust routing in core network is proposed based on the idea of "link criticality" and "path criticality". Betweenness centrality [4], a metric from graph theory, is used to measure the criticality of a link. Suppose that we are measuring the centrality of node $\mathrm{k}$. The betweenness centrality is defined as the share of times a node $\mathrm{i}$ needs a node $\mathrm{k}$ in order to reach a node $\mathrm{j}$ via the shortest path. Link betweenness can also be defined in the same way. A modified version of the link betweenness is used in [3] to define link criticality. Suppose $n_{i j}$ is the number of paths between source-destination pair $(\mathrm{s}, \mathrm{d})$ and $n_{i k j}$ is the number of paths between $\mathrm{i}$, j containing the specific link $\mathrm{k}$. Then betweenness of node $\mathrm{k}$ for $\mathrm{i}$ (source) and $\mathrm{j}$ (destination) is given by $\frac{n_{i k j}}{n_{i j}}$. The overall betweenness of a link is defined 
as the sum of all betweennesses for link $\mathrm{k}$ when $\mathrm{i}, \mathrm{j}$ are changing. This gives an indication of how critical the link is in the network topology. Based on this interpretation of the betweenness centrality, the Path Criticality Routing (PCR) is proposed in [3] to find the least biased paths to run the flow. In [1] the idea of criticality is extended to the DiffServ case, where one has to choose the robust routing plan at the presence of QoS. In [2] a number of graph properties are listed which are useful for determining the robustness of a network against changes in network topology. There is also an abundance of literature for oblivious routing ([5], [6]).

While there are strong results for robustness of the networks, the relationship between the network topology, robustness, congestion, and their interactions is left unattended. In this paper we are trying to answer this question: Is there any specific topology or a class of topologies which are more resilient against congestion? The proposed work is a continuation of our previous research on quantifying the robustness by link and path criticality ([1], [3]). In fact, this work is an analytical description of those results and is justifying the reason behind choosing betweenness as the main metric for criticality measurement and shows how this can be used to achieve robustness at the presence of congestion.

\section{Network Design Problem (NDP)}

In this section we investigate a method for designing the network topologies which are robust according to our definition of robustness.

\section{A. Modeling the behavior of networks with queue-based con- straints}

We start by introducing the notions and metrics. In this paper, we consider the probabilistic definition of the node (link) betweenness as the main metric to quantify the criticality. In [7] a probabilistic interpretation of the betweenness is defined based on random walks. The betweenness of a node (link) $\mathrm{k}$ for source-destination pair $(\mathrm{s}, \mathrm{d})$ is the expected number of times that a random walk passes node $\mathrm{k}$ in its journey from source $\mathrm{i}$ to destination $\mathrm{j}$. The total betweenness of node $\mathrm{k}$ is the sum of this quantity over all possible $(\mathrm{s}, \mathrm{d})$ pairs. Now, the node (link) criticality is simply defined as the random-walk betweenness of that node over its capacity.

$$
\eta_{k}=\frac{b_{k}}{c_{k}}
$$

In equation $1 \eta_{k}, b_{k}, c_{k}$ show the criticality, the betweenness, and the capacity of node $k$ respectively.

The main goal to introduce criticality of the nodes (and links in a similar way) is to be able to sort different networks based on their robustness to the changes in traffic demand, topology, and community of interest (source-destination pairs). Our stress in this section is on the effect of input traffic.

To this end, we consider a network which is shown by its graph $\mathrm{G}=(\mathrm{N}, \mathrm{L})$. Based on our definition of the betweenness, we can quantify the criticality as follows. Each node has a certain probability to send its data to the adjacent nodes. Let's assume a random walk at node $s$ wants to go to node $d$ as its final destination. Destination node is an absorbing state for this random walk and the walk is stopped in destination. The probability for a random-walk previously originated at node $i$ and destined for node $j$ to pass node $k$ in next step is shown by $p_{s k d}$.

$$
p_{s k d}=\left\{\begin{array}{l}
0 \quad \text { if } s=d \\
\sum_{q \in A(s)} w_{s q}
\end{array} \quad\right. \text { otherwise }
$$

where $A(s)$ is the set of adjacent of nodes $s$ and $w_{s k}$ is the weight of link (s, d) if any. The first condition in equation 2 is due to the fact that the destination node $d$ is an absorbing node, and any random-walk coming to this state, will be absorbed or equivalently $b_{d k d}=0$. The betweenness of node (link) $k$ for the source-destination pair $(s, d)$ is shown by $b_{s k d}$ defined as the expected number of times that a random walk from $s$ to $d$ traverses $k$.

Note that the path from $\mathrm{i}$ to $\mathrm{k}$ could be of length 0 to infinity. If we show the probability values $p_{s k d}$ with matrix $P_{d}$, then for all $k \neq d$, the probability of entering node $\mathrm{k}$ at $q^{\text {th }}$ step for different values of $\mathrm{s}$ and $\mathrm{k}$ can be obtained from corresponding members of the matrix $P_{d}^{q}$ and in case of $k=d$ it would be 0 . In our calculations, we treat the destination $d$ as a fixed point and write all matrices based on this assumption. At the end we obtain the general response for our metrics by adding up the results for different destinations. In other words, matrix $P_{d}$ can be viewed as routing matrix to destination $d$ with the property that it starts from node $s$ (rows of the matrix) and traverses node $k$ (columns of the matrix) at least once before reaching at $d$. Hence one can write this relationship in matrix form as follows:

$$
\begin{aligned}
& B_{d}=\left[b_{s k d}\right]=\left\{\begin{array}{l}
\sum_{q=0}^{\infty} P_{d}^{q} \text { if } k \neq d \\
0 \text { otherwise }
\end{array}\right. \\
& B_{d}=\left[b_{s k d}\right]=\sum_{q=0}^{\infty} P_{d}^{q} \times \Theta_{j}
\end{aligned}
$$

where, $b_{s k d}$ is the node betweenness of $\mathrm{k}$ for source-destination pair $(\mathrm{i}, \mathrm{j})$ and $B_{d}$ is it's matrix. Further

$$
\Theta_{j}=\left[\theta_{s k d}\right]=\left\{\begin{array}{ll}
1 & \text { if } s=k \neq d \\
0 & \text { otherwise }
\end{array}\right\}
$$

In words, $\Theta_{d}$ is the Identity Matrix unless its $d^{\text {th }}$ column which is zero. Hence:

$$
B_{d}=\left(\sum_{l=0}^{\infty} P_{d}^{q}\right) \times \Theta_{j}=\left(I-P_{d}\right)^{-1} \times \Theta_{d}
$$

1) Identifying the robustness of a network: In order to determine the robustness of a given topology to the congestion, one needs to know how the traffic is feeding to the network and how the load is being processed. We consider the case that the traffic demands come at the nodes of the network with Poisson rate $\lambda$ and assume that our network is a Jackson network [8]. Based on Jackson's theorem [8], if the input traffic to each one of the nodes is an independent Poisson process and the servers have exponentially distributed service rates, then each node can be modeled by a M/M/1 queue. Every node has a 


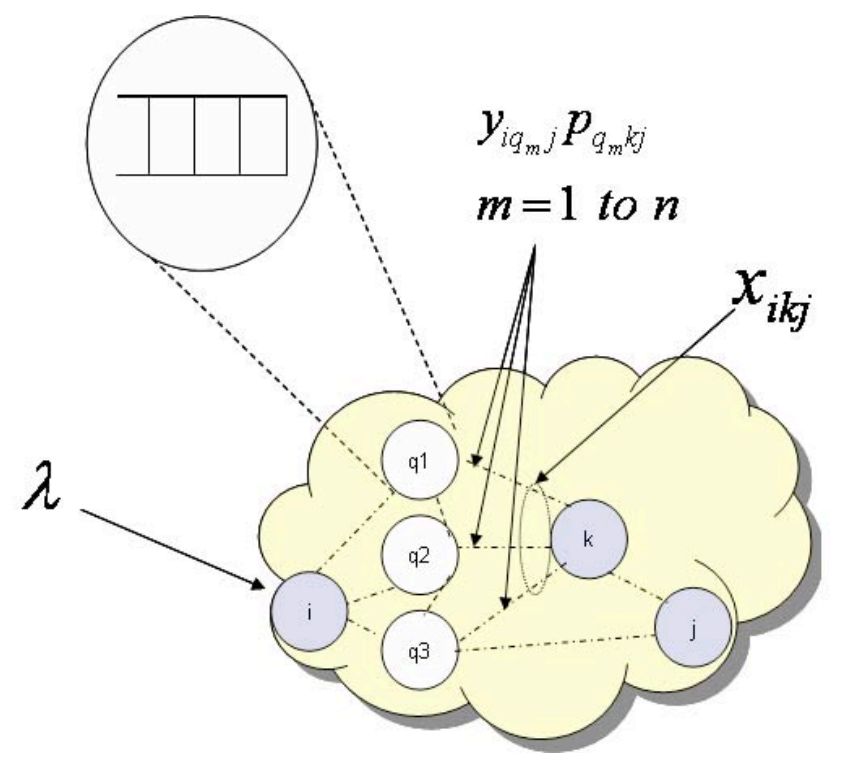

Fig. 1. Network of queues

limited processing power and can process input traffic at the rate of $\mu$ and the traffic demands that are not serviced yet, are queued. We need to find the range of input rate $\lambda$ that keeps the queue in stable condition (in other words we need to rule out the invalid values of $\lambda$ that make the $\mathrm{M} / \mathrm{M} / 1$ queue unstable according to Jackson's theorem). To this end, we calculate the total arrival/departure rate of the traffic to/from each node. The steady state input rate of the messages into the node $s$ destined for $d$ via node $\mathrm{k}$ is assumed to be $x_{s k d}$ and it is equal to the probabilistic share of each and every other node in $s$ in addition to the share of external traffic in node $s$ (for destination $d$ via node $\mathrm{k}$ ). We also assume the total output rate of each node $s$ to destination $d$ via node $\mathrm{k}$ is $y_{s k d}$ (which is equal to $x_{s k d}$ in steady state). The share of every other node on the stationary input rate of $\mathrm{s}$ (for $\mathrm{d}$ through $\mathrm{k}$ ) is $\sum_{t=1}^{n} y_{s t d} \times p_{t k d}$ (Figure 1).

Considering the external traffic rate of $\lambda$ at node $s$, on average $\kappa_{s}$ is passing through node $\mathrm{k}$ to its destination node $d$. Hence, one can write the stationary arrival rate of traffic to node $k$ as follows (bearing in mind that in steady state $\left.y_{s k d}=x_{s k d}\right)$ :

$$
x_{s k d}=\left\{\begin{array}{l}
\sum_{t=1}^{n} x_{s t d} \times p_{t k d}+\kappa_{s} \quad \text { ifs }=k \neq d \\
0 \text { otherwise }
\end{array}\right.
$$

in matrix form:

$$
\begin{aligned}
&\left.X_{d}=\left(\begin{array}{ccccc}
\kappa_{1} & 0 & 0 & 0 & 0 \\
0 & \kappa_{2} & 0 & 0 & 0 \\
0 & 0 & . & 0 & 0 \\
0 & 0 & 0 & . & 0 \\
0 & 0 & 0 & 0 & \kappa_{n}
\end{array}\right)+X_{d} \times P_{d}\right) \times \Theta_{d} \\
& \text { so: } X_{d} \times\left(I-P_{d} \times \Theta\right)=\left(\begin{array}{ccccc}
\kappa_{1} & 0 & 0 & 0 & 0 \\
0 & \kappa_{2} & 0 & 0 & 0 \\
0 & 0 & . & 0 & 0 \\
0 & 0 & 0 & . & 0 \\
0 & 0 & 0 & 0 & \kappa_{n}
\end{array}\right) \times \Theta_{j}
\end{aligned}
$$

But $\kappa_{s}$ is the share of average input rate $\lambda$ on node $s$, which can be written as the product of $\lambda$ and the stationary probability of node $s$ shown by $\pi_{s}$. This means that:

$$
\begin{gathered}
\left(\begin{array}{ccccc}
\kappa_{1} & 0 & 0 & 0 & 0 \\
0 & \kappa_{2} & 0 & 0 & 0 \\
0 & 0 & . & 0 & 0 \\
0 & 0 & 0 & . & 0 \\
0 & 0 & 0 & 0 & \kappa_{n}
\end{array}\right)=\lambda \times\left(\begin{array}{ccccc}
\pi_{1} & 0 & 0 & 0 & 0 \\
0 & \pi_{2} & 0 & 0 & 0 \\
0 & 0 & . & 0 & 0 \\
0 & 0 & 0 & . & 0 \\
0 & 0 & 0 & 0 & \pi_{n}
\end{array}\right) \\
\text { finally: } \quad X_{d}=\lambda \times \Pi \times \Theta_{d} \times\left(I-P_{d} \times \Theta_{d}\right)^{-1}
\end{gathered}
$$

where matrix $\Pi$ represents the diagonal matrix with $\left[\begin{array}{llll}\pi_{1} & \pi_{2} & \ldots & \pi_{n}\end{array}\right]$ as its main diagonal.

To find the relationship of betweenness $B$ and the input arrival rate $\mathrm{X}$ we notice that $P_{d}=\Theta_{d} \times P_{d}$ since $p_{d k d}=0$. Hence:

$$
\begin{gathered}
\Theta_{d} \times\left(I-P_{d} \times \Theta_{d}\right)=\left(I-P_{d}\right) \times \Theta_{d} \\
\text { or } \quad \Theta_{d} \times\left(I-P_{d} \times \Theta_{d}\right)^{-1}=\left(I-P_{d}\right)^{-1} \times \Theta_{d} \\
\text { this means that: } \quad X_{d}=\lambda \times \Pi \times B_{d}
\end{gathered}
$$

Equation 4 asserts that

$$
x_{s k d}=\lambda \times \pi_{s} \times b_{s k d}
$$

Now we use the fact that the stationary probability of any node $s$ in a Markovian network is equal to the normalized betweenness of that node:

$$
\pi_{s}=\frac{b_{s}}{\sum_{i} b_{i}}
$$

Replacing equation 6 in equation 5 results in

$$
x_{s k d}=\lambda \times \frac{b_{s}}{\sum_{i} b_{i}} \times b_{s k d}
$$

To obtain total input rate at any node $k$ one can write

$$
\begin{aligned}
x_{k} & =\sum_{s} \sum_{d} x_{s k d} \\
& =\sum_{s} \sum_{d} \lambda \frac{b_{s}}{\sum_{i} b_{i}} \times b_{s k d} \\
& =\frac{\lambda}{\sum_{i} b_{i}} \times \sum_{s} b_{s} \sum_{d} b_{s k d} \\
& \leq \frac{\lambda}{\sum_{i} b_{i}} \times b_{\max } \times \sum_{s} \sum_{d} b_{s k d} \\
& =\frac{\lambda}{\sum_{i} b_{i}} \times b_{\max } \times b_{k}
\end{aligned}
$$

where $b_{\max }$ is the maximum node betweenness in the graph. Now we calculate the average input rate for all the nodes.

$$
\begin{aligned}
\bar{x} & =\frac{\sum_{k} x_{k}}{n} \\
& \leq \frac{1}{n} \times \frac{\lambda}{\sum_{i} b_{i}} \times b_{\max } \times \sum_{k} b_{k} \\
& =\frac{\lambda}{n} \times b_{\max } \\
\bar{x} & \leq \frac{\lambda}{n} \times b_{\max }
\end{aligned}
$$


If the service rate of any node queue be $\mu$ and the capacity of a node $k$ be $c_{k}\left(c_{k}\right.$ is the sum of the capacity of the links incident to the node $k$ ), considering equation 8

$$
\begin{aligned}
\max (\bar{x}) & \leq \mu c^{*} \\
\frac{\lambda}{n} \times b_{\max } & \leq \mu c^{*} \\
\frac{\lambda}{\mu} & \leq \frac{n \times c^{*}}{b_{\max }} \\
\frac{\lambda}{\mu} & \leq \frac{n}{\left(\frac{b_{\max }}{c^{*}}\right)} \\
\frac{\lambda}{\mu} & \leq \frac{n}{\eta_{\max }}
\end{aligned}
$$

$c^{*}$ in equation 9 shows the capacity of the node whose betweenness is maximum amongst all the nodes. Furthermore, according to our definition of criticality, $\frac{b_{\max }}{c^{*}}$ in equation 10 is indeed the maximum node criticality of the graph $\left(\eta_{m} a x\right)$. We can summarize these results in theorems 3.1, and 3.2.

Theorem 3.1: Consider a network topology $\mathrm{G}(\mathrm{N}, \mathrm{L})$, and assume there is external expected input traffic rate of $\lambda$ for each node of the network. Let $x_{\max }$ be the maximum expected arrival rate of all the nodes $b_{\max }$ be the total betweenness of the node with maximum expected input rate, then:

$$
x_{\max }=\frac{\lambda}{n} \times b_{\max }
$$

Theorem 3.2: To maximize the offered load of a network, one needs to minimize the maximum criticality of all the nodes in the network.

Proof: The proof is a direct result of equation 10. According to this equation, the offered load $\frac{\lambda}{\mu}$ is proportional to the reciprocal of the maximum node criticality. This means that to maximize $\frac{\lambda}{\mu}$ one needs to minimize $\eta_{\max }$.

The importance of theorem 3.2 lies in the fact that it proposes an optimization problem which is suitable for network topology design and shows the direct relationship between the topology of the network and the network robustness against congestion. While theorem 3.2 provides a powerful framework for network design problem (NDP), it needs more mathematical work to extract the attributes and behavior of the criticality function, which is beyond the scope of this paper and will be addressed in our future research papers.

If we assume that the capacity of the nodes are fixed, then according to equation 9, a similar optimization problem based on the analysis of the node betweenness can be extracted. In next section we investigate the behavior of node betweenness in more details and obtain two different optimization problems to characterize the robustness of a network.

2) Analysis: As it is clear from the previous section, one needs to satisfy $x_{k}<x^{m}$ condition to avoid congestion. The best topology is the one that maximizes the marginal input rate $x^{m}$ or equivalently minimizes the maximum node (or link) betweenness $b^{m}$ which is an optimization problem. In order to analyze the system behavior, one needs to know the reaction of the control system to the changes in input rate $\lambda$. Two boundary conditions are considered, $\lambda \rightarrow 0^{+}$and $\lambda \rightarrow \lambda^{m-}$ (congestion mode). In order to analyze the system behavior in these conditions, we first derive the average queue length in each one of the nodes and the total number of waiting messages in the whole system. According to the queueing theory [8] , the average length of the $\mathrm{M} / \mathrm{M} / 1$ queue in node $\mathrm{p}$ is:

$$
\bar{l}_{p}=\frac{\rho_{p}}{1-\rho_{p}}=\frac{\frac{x_{p}}{\mu}}{1-\frac{x_{p}}{\mu}}=\frac{\frac{\lambda \times b_{p}}{n}}{1-\frac{\lambda \times b_{p}}{n}}
$$

where we assumed $\mu=1$. Now, the total queue length in the whole network is:

$$
\bar{L}=\sum_{p \in N} \bar{l}_{p}=\sum_{p \in N} \frac{\frac{\lambda \times b_{p}}{n}}{1-\frac{\lambda \times b_{p}}{n}}
$$

a) $\lambda \rightarrow 0^{+}$

In this situation the total queue length can be approximated by

$$
\bar{L} \simeq \sum_{p \in N} \frac{\lambda \times b_{p}}{n}=\frac{\lambda}{n} \times \sum_{p \in N} b_{p}=\lambda \times \bar{b}
$$

This result shows that, when the external input traffic rate is small, one needs to minimize the average node betweenness of the graph.

b) $\lambda \rightarrow \lambda^{m-}$

The total queue length in this case would be:

$$
\bar{L} \simeq \max \left(\frac{1}{1-\frac{\lambda \times b_{p}}{n}}\right)=\frac{1}{1-\frac{\lambda}{n} \times \max \left(b_{p}\right)}
$$

Clearly, when the external traffic rate is close to the marginal value (congestion mode), in order to minimize the total queue length, one needs to minimize the maximum betweenness among all nodes. The results are summarized in theorem 3.3.

Theorem 3.3: In a network G(N,L) with Poisson distribution and rate $\lambda$, let each node be modeled with an $\mathrm{M} / \mathrm{M} / 1$ queue, then to minimize the congestion, one needs to minimize the maximum node/link betweenness (or equivalently the topological node/link criticality) of the graph $G(N, L)$. Further, when the load is low, the average graph betweenness should be minimized to achieve the best goodness.

Theorem 3.3 suggests that the maximum node betweenness and minimum node betweenness of a topology can be used to quantify the robustness of topology to the congestion. We choose to have the maximum deviation of the node betweenness as our main congestion robustness metric.

We define the network robustness index (NRI) as follows:

$$
N R I(G)=\sum_{i=1}^{n}\left(B_{i}-\bar{B}\right)^{2}
$$




\section{Evaluation}

In this section we investigate different network topologies and experience the effect of topology in NRI of a network.

\section{A. Star Network versus Full Mesh}

We begin with star network as the most centralized topology. star network has the least average node betweenness among the set of all graphs that can be built on $\mathrm{N}$ nodes. It is not difficult to show that:

$$
N R I(\text { Star })=\frac{n}{n-1}
$$

For large networks the NRI for star network approaches unity. For complete network or full mesh which is the most decentralized topology (in contrast to star which is the most centralized one), the network robustness index is zero. These are two extremes for NRI. While star network has the highest NRI and lowest average node criticality (betweenness), the full-mesh has the lowest NRI with the same average criticality. As a result, one reasonable design guideline for designing appropriate network topologies is to move towards a complete graph (or at least regular graph) when the network is working near the congestion point most of the time, while the centralized version is a suitable choice for situations that the network is small and its working point (in terms of traffic) is far from congestion. In brief one can say near complete graphs networks are appropriate for core or backbone while star topology is useful for edge.

\section{B. Simple 4-node network}

Fig. 2 shows four networks built on 4 nodes starting from star and ending with complete network on 4 nodes.
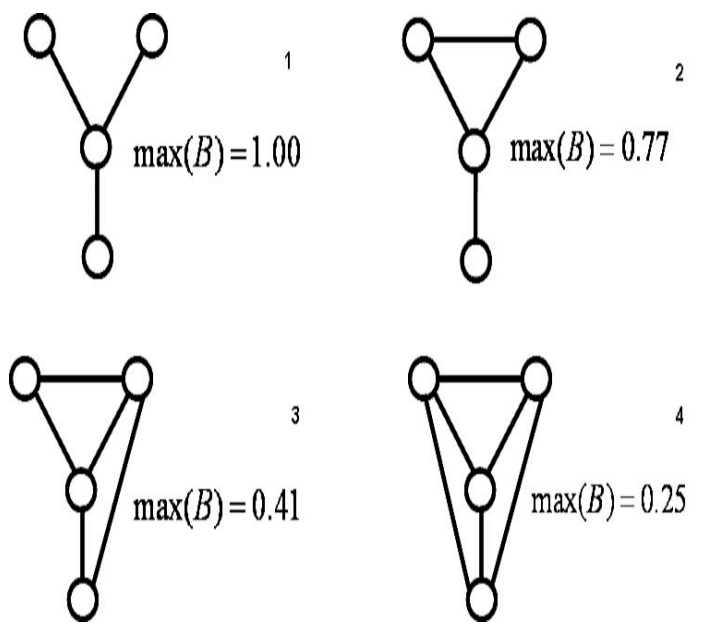

Fig. 2. Maximum node betweenness for 4-node topologies

\section{Abilene network}

Our third example is the Abilene network [9]. The topology of Abilene network is shown in Fig. 4. We have examined the Abilene network. The betweenness of all the nodes are listed in table (a). Node 5 has the maximum betweenness which is equal to 0.391 . The average betweenness of the network is 0.252 and the network robustness index is $N R I$ (Abilene $)=$ 0.112. Comparing with star topology there is great improvement but still a little far from the optimal case which is the complete graph with $\mathrm{NRI}=0$.

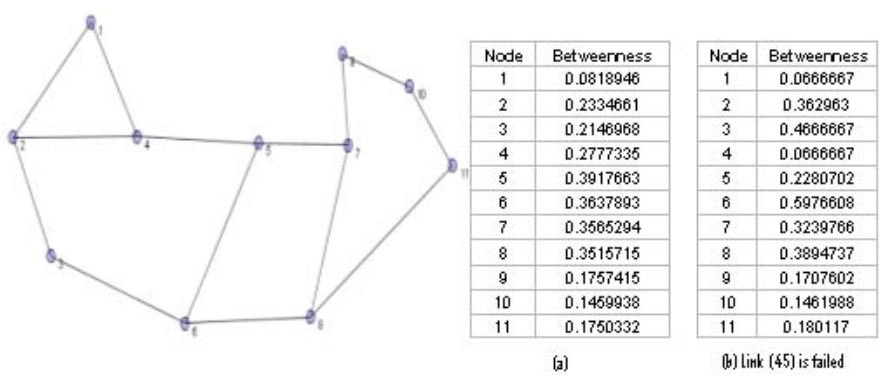

Fig. 3. Abilene Network Topology

In order to investigate the effect of link failure on the robustness of the network, we failed link $(4,5)$ and measured the betweenness for the reduced topology (note that the network is still connected). The new node betweennesses are shown in table (b) of Fig. 4. In the new configuration the maximum betweenness is 0.598 and belongs to node 6 . The average node betweenness is 0.273 and the network robustness index is $N R I($ Abilene 1$)=0.273$. This result is expected since the removal of link $(4,5)$ destroys some of the paths between end nodes and put more traffic on the rest of the network.

\section{Random Graphs}

We finally examined the network robustness index of random graphs. We used random graphs of type Erdos-Renyi [10] with different number of nodes and probability for the links. The result show that for $n>8$ and $p>.5$ ( $\mathrm{p}$ is the probability of having a link between two nodes), the NRI was always less than 0.03. This is in line with the results of [2]. In [2] has been shown that random graphs are more robust to the network failure. Our results show that random networks are also more resilient against congestion in the network. In Fig. 4 an Erdos-Rrnyi random graph with 10 nodes and probability of 0.9 for creating a link between two nodes is shown. The random-walk betweenness of the nodes are also shown. It can be seen that the betweenness is fairly uniform (although not equal) among the nodes and the network robustness index is $\mathrm{NRI}=0.02$.

\section{E. NRI in Practical Networks}

This section provides the result of measurements on a practical network shown in Fig. 5. We started our experiment by measuring the average betweenness and NRI for this network. The average betweenness was 0.1497 and the NRI was 0.0065 . This result is shown in the first row of the table of Fig. 6. The NRI suggests that the network robustness should be good. To evaluate the robustness, we consecutively failed links of the network one at a time and measured the 

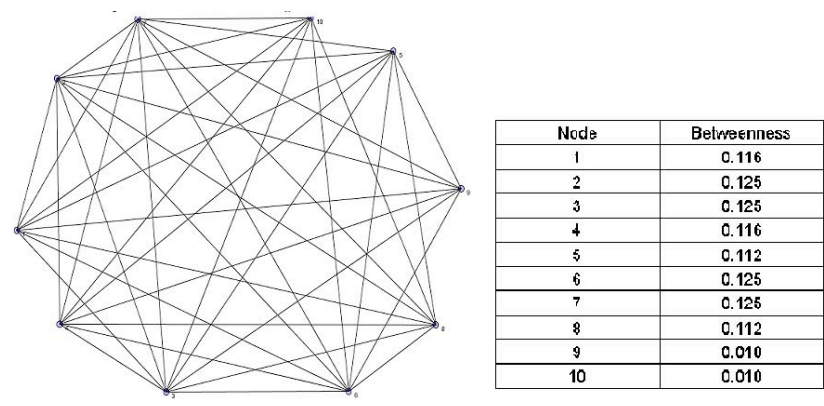

Fig. 4. Erdos-Renyi Random graph: $n=10, p=0.9$
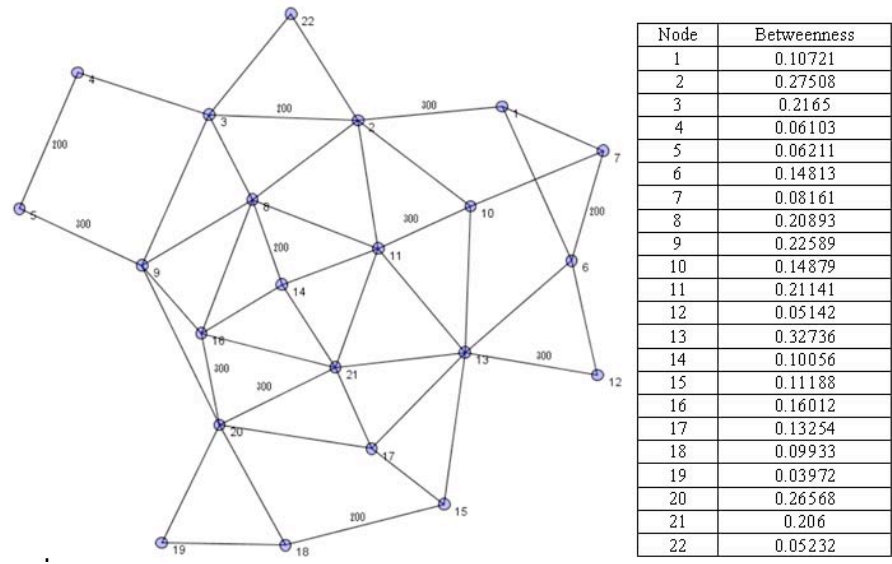

Fig. 5. Test Network

average betweenness and NRI for reduced networks in each step. Each line of the table of Fig. 6 shows one step including the failed link (column 1), the average betweenness (column 2 ) and NRI (column 3). The results are also plotted in a graph as well. The graph shows that the network is fairly robust to the link failure and its NRI does not have a big dramatic increase in first 10 link removal ana after that starts increasing.

\section{CONCLUSION}

We investigated the effect of network topology on the congestion of networks and proposed an analytical approach to the network design problem (NDP) with the goal of having more robust network topology. We used the variance of the

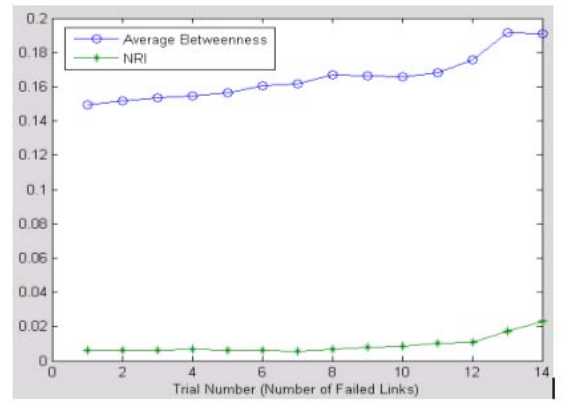

\begin{tabular}{|c|c|c|}
\hline Removed & Mean(B) & NRI \\
\hline Initial & 0.1497 & 0.0064 \\
\hline$(2,3)$ & 0.1518 & 0.0062 \\
\hline$(3,8)$ & 0.1536 & 0.0064 \\
\hline$(8,11)$ & 0.1548 & 0.0065 \\
\hline$(11,13)$ & 0.1567 & 0.0063 \\
\hline$(13,21)$ & 0.1605 & 0.0059 \\
\hline$(21,20)$ & 0.1617 & 0.0058 \\
\hline$(20,9)$ & 0.167 & 0.007 \\
\hline$(9,8)$ & 0.1665 & 0.008 \\
\hline$(8,14)$ & 0.1659 & 0.0084 \\
\hline$(14,21)$ & 0.1682 & 0.0104 \\
\hline$(21,17)$ & 0.1757 & 0.0109 \\
\hline$(17,20)$ & 0.1914 & 0.0171 \\
\hline$(20,16)$ & 0.1908 & 0.023 \\
\hline
\end{tabular}

Fig. 6. Test of Network Sensitivity to the Link Failure node betweenness array of the graph as the network robustness index (NRI) and ranked different networks with their NRI, starting from complete graph with $N R I=0$ to the star with $N R I \approx 1$.

The problem of capacity assignment is also considered and a proposal is developed for the optimal assignment of capacities using the concept of effective resistance of a graph.

There are different venues for further investigation. One possible future work is to integrate the results of our investigation into a consolidated framework for the first phase of network management which is the design of a robust network topology and to maintain the appropriate properties of the network (such as low betweenness, good connectivity) by developing appropriate algorithms. Another research proposal is to explore the effect of connectivity as one of the critical features of a network topology and to develop a procedure to design well-connected networks.

\section{REFERENCES}

[1] A. Tizghadam and A. Leon-Garcia. A robust routing plan to optimize throughput in core networks. ITC20, Elsvier, pages 117-128, 2007.

[2] A. H. Dekker and B. D. Colbert. Network robustness and graph topology. Australasian Computer Science Conference, 26:359-368, Jan. 2004.

[3] A. Tizghadam and A. Leon-Garcia. Lsp and back up path setup in mpls networks based on path criticality index. ICC 2007, IEEE, pages 441-448, 2007.

[4] L. C. Freeman. Centrality in networks: I. conceptual clarification. Social Networks, (1):215-239, 1978/79.

[5] M. S. Kodialam and et al. Maximum throughput routing of traffic in the hose model. INFOCOM, pages 1217-1225, 2006.

[6] N. G. Duffield and et al. A flexible model for resource management in virtual private network. ACM SIGCOMM, Aug. 1999.

[7] M. Newman. A measure of betweenness centrality based on random walks. arXiv cond-mat/0309045., 2003.

[8] A. Leon-Garcia. "Probability and Random Processes for Electrical Engineering”. Addisn Wesley, 2th edition, 1993.

[9] www.internet2.org.

[10] B. Bollobs. "Random Graphs". Cambridge University Press, 2th edition, 2001. 